\title{
Neoantigen Vaccine GEN-009
}

National Cancer Institute

\section{Source}

National Cancer Institute. Neoantigen Vaccine GEN-009. NCI Thesaurus. Code C162874.

A peptide-based, personalized cancer vaccine consisting of patient-specific mutated synthetic long peptides (SLPs), which are immunogenic and unique to the patient's tumor, with potential immunomodulating and antineoplastic activities. Upon subcutaneous vaccination with the personalized neoantigen peptide vaccine GEN-009, and administration along with the immunoadjuvant poly-ICLC, the peptides stimulate the host immune system to mount a specific and potent cytotoxic T-lymphocyte (CTL) response against tumor cells expressing the neoantigens, which results in tumor cell lysis. The Antigen Lead Acquisition System (AT LAS) will be used to identify neoantigens in each patient's tumor that are recognized by their CD4 and/or CD8 T-cells and will be incorporated into GEN-009 in the form of SLPs. Each personalized vaccine contains between four and twenty SLPS. 